\title{
Triple Differential Cross-Sections for Ionization of H(3d) by Incident Electron
}

\author{
Sushmita Banerjee ${ }^{*}$, Sunil Dhar ${ }^{1}$, Amanul Hoque $^{2}$ \\ ${ }^{1}$ Department of Mathematics, Chittagong University of Engineering and Technology, Chittagong, Bangladesh \\ ${ }^{2}$ Faculty of Business Administration, BGC Trust University, Chittagong, Bangladesh \\ Email: *sushmita@cuet.ac.bd
}

How to cite this paper: Banerjee, S., Dhar, S. and Hoque, A. (2018) Triple Differential Cross-Sections for Ionization of $\mathrm{H}(3 \mathrm{~d})$ by Incident Electron. Open Journal of Microphysics, 8, 30-41.

https://doi.org/10.4236/ojm.2018.84005

Received: November 7, 2018

Accepted: November 27, 2018

Published: November 30, 2018

Copyright (c) 2018 by authors and Scientific Research Publishing Inc. This work is licensed under the Creative Commons Attribution International License (CC BY 4.0).

http://creativecommons.org/licenses/by/4.0/

\begin{abstract}
Triple differential cross sections (TDCS) are estimated for the ionization of metastable 3d-state hydrogen atoms by electron at $250 \mathrm{eV}$ for various kinematic conditions pursuing a multiple scattering theory. The present new results are compared with the theoretical results of hydrogenic different metastable states as well as the hydrogenic ground state experimental data. Obtained new finding results are in good qualitative agreement with those of compared theories. The present results give an immense opportunity for experimental trial in the field of ionization problems.
\end{abstract}

\section{Keywords}

Electron, Ionization, Cross-Sections, Scattering

\section{Introduction}

The theoretical non-relativistic studies for the atomic ionization by fast particle were first treated by Bethe [1]. Electron impact ionization by electrons processes is used in a diverse range of fields, such as radiation physics, plasma physics as well as astrophysics. Over the last four decades, the theoretical and experimental study in electron atom ionization collision on different cross sections has become progressively interesting for non-relativistic [2]-[29] as well as relativistic [30] [31] [32] [33] [34] energies. The atomic hydrogen ionization by fast particle is a good form for perturbation theory due to the availability of experimental results. In this context, the electron-electron coincidence experiments called (e, 2e) experiments, provide a clear concept of the kinematics of the collisions by giving information about the direction of the scattered and ejected electrons. During the last five decades, ionization of hydrogen atom by electron has been considered to explore the details of the ionization process both in the ground 
state [2]-[15] and metastable states [16]-[29] of atomic hydrogen.

In the field of electron, impact ionization is to develop a general theoretical framework, which will provide the accurate ionization cross sections for many atoms over a practically relevant impact energy range. Due to its perplexity, the fully quantum mechanical treatment of atomic ionization by electron is possible for the artless cases of hydrogen atom. In this work, atomic hydrogen is used as target in order to perceive the ionization mechanism of atomic system by electron impact energy.

Hydrogenic metastable $3 \mathrm{~d}$ state is an excited state which has a relatively long lifetime than the other excited states. A metastable sate has a higher energy than the ground state. The lifetime of excited state is given by [35]

$$
T_{i}=\left(\sum A_{i j}\right)^{-1}
$$

where $A_{i j}$ is Einstein A coefficient. The lifetime of metastable 3d state of hydrogen atom is $2.3 \times 10^{-7} \mathrm{~s}$.

A multiple scattering theory [5] [15] has been applied in the present calculation of the triple differential cross sections (TDCS) in the metastable $3 \mathrm{~d}$-state hydrogen atom ionization by $250 \mathrm{eV}$ electron energy. Lewis integral [36] has been used in the present study for analytical calculation.

The existent new study results will present a new dimension on ionization of hydrogenic metastable states. Current results are compared with previous related theories [18] [27] and [29].

\section{Theory}

Electron-impact ionization cross section is estimated by taking the ratio of the number of ionization elements per unit time and per unit target to the incident electron flux.

Ionization of atomic hydrogen by electron in most elaborate form is presently available of following type

$$
\mathrm{e}^{-}+\mathrm{H}(3 \mathrm{~d}) \rightarrow \mathrm{H}^{+}+2 \mathrm{e}^{-}
$$

Here $3 \mathrm{~d}$ denotes the hydrogenic metastable state and has been attained in the coplanar geometry by examining TDCS measured in $(e, 2 e)$ coincidence experiments.

The triple differential cross section is denoted by the symbol $\frac{d^{3} \sigma}{d \Omega_{1} d \Omega_{2} d E_{1}}$.

Finally, the total ionization cross section is obtained by integrating over all outgoing scattering angles and energies, and depends only on $E_{i}$, the incident electron energy.

The direct T-matrix element for ionization of hydrogen atoms by electrons, following Das and Seal [15] may be written as

$$
T_{f i}=\left\langle\Psi_{f}^{(-)}\left(\overline{r_{1}}, \overline{r_{2}}\right)\left|V_{i}\left(\overline{r_{1}}, \overline{r_{2}}\right)\right| \Phi_{i}\left(\overline{r_{1}}, \overline{r_{2}}\right)\right\rangle
$$

here, $\bar{r}_{1}$ and $\bar{r}_{2}$ represent the coordinates of the atomic active electron and the 
incident electron, $\left(\bar{p}_{1}, \bar{p}_{2}\right)$ and $\left(E_{1}, E_{2}\right)$ represent the momenta and energies of the two electrons in the final state and $\left(\bar{p}_{i}, E_{i}\right)$ are the momentum and the energy of the incident electron.

Where the perturbation potential $V_{i}\left(\bar{r}_{1}, \bar{r}_{2}\right)$ is given by

$$
V_{i}\left(\bar{r}_{1}, \bar{r}_{2}\right)=\frac{1}{r_{12}}-\frac{Z}{r_{2}}
$$

The nuclear charge of the hydrogen atom is $Z=1, r_{1}$ and $r_{2}$ are the distance of the two electrons from the nucleus and $r_{12}$ is the distance between two electrons.

We have the initial channel unperturbed wave function is

$$
\Phi_{i}\left(\bar{r}_{1}, \bar{r}_{2}\right)=\frac{\mathrm{e}^{\overline{\bar{p}_{i}} \cdot \overline{\bar{r}_{2}}}}{(2 \pi)^{3 / 2}} \phi_{3 d}\left(\bar{r}_{1}\right) .
$$

where

$$
\begin{gathered}
\phi_{3 d}\left(\bar{r}_{1}\right)=\frac{1}{81 \sqrt{6 \pi}}\left(r_{1}^{2}\right)\left(3 \cos ^{2} \theta-1\right) \mathrm{e}^{-r_{1} / 3}=\frac{1}{324 \sqrt{3} \pi^{2}}\left(r_{1}^{2}\right)\left(3 \cos ^{2} \theta-1\right) \mathrm{e}^{-\lambda_{1} r_{1}} . \\
\Phi_{i}\left(\bar{r}_{1}, \bar{r}_{2}\right)=\frac{1}{324 \sqrt{3} \pi^{2}}\left(r_{1}^{2}\right)\left(3 \cos ^{2} \theta-1\right) \mathrm{e}^{-\lambda_{1} r_{1}}
\end{gathered}
$$

here $\lambda_{1}=\frac{1}{3}, \phi_{3 d}\left(\bar{r}_{1}\right)$ is the hydrogenic $3 \mathrm{~d}$-state wave function and $\Psi_{f}^{(-)}\left(\bar{r}_{1}, \bar{r}_{2}\right)$ is approximate wave function is given by [15]

$$
\begin{aligned}
& \Psi_{f}^{(-)}\left(\bar{r}_{1}, \bar{r}_{2}\right)=N\left(\bar{p}_{1}, \bar{p}_{2}\right)\left[\phi_{\bar{p}_{1}}^{(-)}\left(\overline{r_{1}}\right) \mathrm{e}^{\overline{p_{2}} \cdot \bar{r}_{2}}+\phi_{\bar{p}_{2}}^{(-)}\left(\bar{r}_{2}\right) \mathrm{e}^{i \bar{p}_{1} \cdot \overline{r_{1}}}\right. \\
& \left.+\phi_{\bar{p}}^{(-)}(\bar{r}) \mathrm{e}^{i \bar{P} \cdot \bar{R}}-2 \mathrm{e}^{i \overline{p_{1}} \cdot \overline{\eta_{1}}+\bar{p}_{2} \cdot \bar{p}_{2}}\right] /(2 \pi)^{3}
\end{aligned}
$$

where

$$
\begin{array}{cc}
\bar{r}=\frac{\bar{r}_{2}-\bar{r}_{1}}{2}, \quad \bar{R}=\frac{\bar{r}_{1}+\bar{r}_{2}}{2}, \\
\bar{p}=\left(\bar{p}_{2}-\bar{p}_{1}\right), \quad \bar{P}=\bar{p}_{2}+\bar{p}_{1},
\end{array}
$$

The normalization constant $N\left(\bar{p}_{1}, \bar{p}_{2}\right)$ is given by

$$
\begin{aligned}
\left|N\left(\bar{p}_{1}, \bar{p}_{2}\right)\right|^{-2}= & \mid 7-2\left[\lambda_{1}+\lambda_{2}+\lambda_{3}\right]-\left[\frac{2}{\lambda_{1}}+\frac{2}{\lambda_{2}}+\frac{2}{\lambda_{3}}\right] \\
& +\left[\frac{\lambda_{1}}{\lambda_{2}}+\frac{\lambda_{1}}{\lambda_{3}}+\frac{\lambda_{2}}{\lambda_{1}}+\frac{\lambda_{2}}{\lambda_{3}}+\frac{\lambda_{3}}{\lambda_{1}}+\frac{\lambda_{3}}{\lambda_{2}}\right] \mid
\end{aligned}
$$

where

$$
\begin{aligned}
& \lambda_{1}=\mathrm{e}^{\pi \alpha_{1} / 2} \Gamma\left(1-i \alpha_{1}\right), \quad \alpha_{1}=\frac{1}{p_{1}} \\
& \lambda_{2}=\mathrm{e}^{\pi \alpha_{2} / 2} \Gamma\left(1-i \alpha_{2}\right), \quad \alpha_{2}=\frac{1}{p_{2}} \\
& \lambda_{3}=\mathrm{e}^{\pi \alpha / 2} \Gamma(1-i \alpha), \quad \alpha=-\frac{1}{p}
\end{aligned}
$$

the Coulomb wave function $\phi_{q}^{(-)}(\bar{r})$ is given by 


$$
\phi_{q}^{(-)}(\bar{r})=\mathrm{e}^{\pi \alpha / 2} \Gamma(1+i \alpha) \mathrm{e}^{i q \cdot \bar{r}} F_{1}(-i \alpha, 1,-i[q r+\bar{q} \cdot \bar{r}])
$$

with

$$
\alpha_{1}=\frac{1}{p_{1}} \text { for } \bar{q}=\bar{p}_{1}, \quad \alpha_{2}=\frac{1}{p_{2}} \text { for } \bar{q}=\bar{p}_{2}
$$

and

$$
\alpha=-\frac{1}{p} \text { for } \bar{q}=\bar{p}
$$

Now Equation (2) becomes

$$
T_{f i}=T_{B}+T_{B^{\prime}}+T_{i}-2 T_{P B}
$$

where

$$
\begin{aligned}
& T_{B}=\left\langle\phi_{p_{1}}^{(-)}\left(\overline{r_{1}}\right) \mathrm{e}^{i \overline{p_{2}} \cdot \overline{r_{2}}}\left|V_{i}\right| \Phi_{i}\left(\overline{r_{1}}, \overline{r_{2}}\right)\right\rangle \\
& T_{B^{\prime}}=\left\langle\phi_{p_{2}}^{(-)}\left(\overline{r_{2}}\right) \mathrm{e}^{i \bar{p}_{1} \cdot \bar{r}_{1}}\left|V_{i}\right| \Phi_{i}\left(\bar{r}_{1}, \bar{r}_{2}\right)\right\rangle \\
& T_{i}=\left\langle\phi_{p}^{(-)}(\bar{r}) \mathrm{e}^{i \bar{P} \cdot \bar{R}}\left|V_{i}\right| \Phi_{i}\left(\bar{r}_{1}, \bar{r}_{2}\right)\right\rangle \\
& T_{P B}=\left\langle\mathrm{e}^{i \bar{p}_{1} \cdot \bar{r}_{1}+\bar{p}_{2} \cdot \bar{r}_{2}}\left|V_{i}\right| \Phi_{i}\left(\bar{r}_{1}, \bar{r}_{2}\right)\right\rangle
\end{aligned}
$$

here Equation (6) is called First Born term and it may be written as

$$
\begin{aligned}
& T_{B}=\frac{1}{324 \sqrt{3} \pi^{2}}\left\langle\phi_{p_{1}}^{(-)}\left(\overline{r_{1}}\right) \mathrm{e}^{\overline{\bar{p}_{2}} \cdot \bar{r}_{2}}\left|\frac{1}{r_{12}}-\frac{1}{r_{2}}\right| \mathrm{e}^{i \bar{p}_{i} \cdot \bar{r}_{2}}\left(r_{1}^{2}\right)\left(3 \cos ^{2} \theta-1\right) \mathrm{e}^{-r_{1} \lambda_{1}}\right\rangle \\
& =\frac{1}{324 \sqrt{3} \pi^{2}} \int \phi_{p_{1}}^{(-) *}\left(\overline{r_{1}}\right) \mathrm{e}^{-\overline{p_{2}} \cdot \cdot \cdot \bar{r}_{2}}\left(\frac{1}{r_{12}}-\frac{1}{r_{2}}\right) \mathrm{e}^{\overline{p_{i}} \cdot \cdot \bar{r}_{2}}\left(r_{1}^{2}\right)\left(3 \cos ^{2} \theta-1\right) \mathrm{e}^{-\lambda_{1} r_{1}} \mathrm{~d}^{3} r_{1} \mathrm{~d}^{3} r_{2} \\
& T_{B}=\frac{1}{324 \sqrt{3} \pi^{2}} \int \phi_{p_{1}}^{(-) *}\left(\bar{r}_{1}\right) \mathrm{e}^{-i \bar{p}_{2} \cdot \bar{r}_{2}} \frac{1}{r_{12}} \mathrm{e}^{\overline{p_{i}} \cdot \cdot \overline{r_{2}}} r_{1}^{2}\left(3 \cos ^{2} \theta-1\right) \mathrm{e}^{-\lambda_{1} r_{1}} \mathrm{~d}^{3} r_{1} \mathrm{~d}^{3} r_{2} \\
& -\frac{1}{324 \sqrt{3} \pi^{2}} \int \phi_{p_{1}}^{(-) *}\left(\overline{r_{1}}\right) \mathrm{e}^{-i \bar{p}_{2} \cdot \bar{r}_{2}} \frac{1}{r_{2}} \mathrm{e}^{\overline{p_{i}} \cdot \cdot \overline{r_{2}}} r_{1}^{2}\left(3 \cos ^{2} \theta-1\right) \mathrm{e}^{-\lambda_{1} \eta} \mathrm{d}^{3} r_{1} \mathrm{~d}^{3} r_{2} \\
& T_{B}=\mathrm{tb} 1+\mathrm{tb} 2
\end{aligned}
$$

where

$$
\begin{aligned}
& \mathrm{tb} 1=\frac{1}{324 \sqrt{3} \pi^{2}} \int \phi_{p_{1}}^{(-) *}\left(\overline{r_{1}}\right) \mathrm{e}^{-\overline{\bar{p}_{2}} \cdot \overline{r_{2}}} \frac{1}{r_{12}} \mathrm{e}^{i \overline{p_{i}} \cdot \overline{\bar{r}_{2}}} r_{1}^{2}\left(3 \cos ^{2} \theta-1\right) \mathrm{e}^{-\lambda_{1} \eta_{1}} \mathrm{~d}^{3} r_{1} \mathrm{~d}^{3} r_{2} \\
& \mathrm{tb} 2=-\frac{1}{324 \sqrt{3} \pi^{2}} \int \phi_{p_{1}}^{(-) *}\left(\overline{r_{1}}\right) \mathrm{e}^{-i \overline{p_{2}} \cdot \overline{r_{2}}} \frac{1}{r_{2}} \mathrm{e}^{\overline{\bar{p}_{i}} \cdot \overline{r_{2}}} r_{1}^{2}\left(3 \cos ^{2} \theta-1\right) \mathrm{e}^{-\lambda_{1} r_{1}} \mathrm{~d}^{3} r_{1} \mathrm{~d}^{3} r_{2}
\end{aligned}
$$

Using the Lewis integral [36], we have evaluated First Born term $T_{B}$ of Equation (7).

Similarly, we have calculated analytically the above Equations (8), (9) and (10) for second Born results using the Lewis integral [36]. After that we have computed the above equations using Gaussian quadrature formula. Finally the triple differential cross-sections for T-Matrix element is given by

$$
\frac{\mathrm{d}^{3} \sigma}{\mathrm{d} \Omega_{1} \mathrm{~d} \Omega_{2} \mathrm{~d} E_{1}}=\frac{p_{1} p_{2}}{p_{i}}\left|T_{f i}\right|^{2}
$$




\section{Results and Discussions}

In this section, We have calculated in this work the triple differential cross-sections (TDCS) at high incident energy $E_{i}=250 \mathrm{eV}$ for various ejected angles $\theta_{1}$ and fixed scattering angles $\theta_{2}$. Triple differential cross sections for ionization of metastable $3 \mathrm{~d}$-state hydrogen atoms by incident electron are presented at different energies. The existent results are compared with the ionization of hydrogen atoms by electrons from ground state theoretical results of $\mathrm{Dal}$ et al. [16], the BBK model of Brauner et al. [11] and the experiment results of Ehrhardt et al. [2]. Also the earlier works on hydrogenic 2S-state [18], 3S-state [27] and recent works on hydrogenic 3P-state [29] ionization results are exhibited here for comparison. The present results of triple differential cross section are presented in the following nine figures where we have designed the electron impact TDCS varying against the angle of ejection $\left(\theta_{1}\right)$ of the ejected electron.

In this study, the ejected angle $\theta_{1}$ varies from $0^{\circ}$ to $360^{\circ}$ considered as horizontal axis where scattering angles $\theta_{2}$ is fixed and referred as vertical axis. The present results of hydrogenic metastable $3 \mathrm{~d}$ state by electron are designed corresponding to the different scattering angles $\theta_{2}=30^{\circ}$ Figure $1(\mathrm{a})$ for ejected electron energies $E_{1}=5,15^{\circ}$ Figure $1(\mathrm{~b}), 25^{\circ}$ Figure $1(\mathrm{c})$ for ejected electron energies $E_{1}=50 \mathrm{eV}$ considering the ejected angle $\theta_{1}$ from $30^{\circ}$ to $100^{\circ}$ and the scattering angle $\theta_{2}=5^{\circ}$ Figure $2(\mathrm{a}), 7^{\circ}$ Figure $2(\mathrm{~b}), 9^{\circ}$ Figure $2(\mathrm{c}), 11^{\circ}$ Figure $3(\mathrm{a}), 15^{\circ}$ Figure $3(\mathrm{~b})$ and $20^{\circ}$ Figure $3(\mathrm{c})$ for ejected electron energies $E_{1}=5 \mathrm{eV}$.

The incident electron energy of $E_{i}=250$ is taken here. In all figures, $\theta_{1}\left(0^{\circ}-150^{\circ}\right)$ and $\phi=0^{\circ}$ is considered as recoil region while $\theta_{1}\left(150^{\circ}-360^{\circ}\right)$ and $\phi=180^{\circ}$ is referred as binary region.

In Figure 1(a), the present results shows a qualitative comparison with the present first Born result (black), the hydrogenic ground state result of BBK model [11], the second Born approximation [16], the hydrogenic ground state experimental data [2], 3S-state results [27] and recent works on hydrogenic 3P-state [29] ionization results. The peak values of present results and first Born results show good qualitative agreement with those of the compared results in the recoil region but show somewhere dissimilar in the binary region. This may be happened due to the change of the hydrogenic metastable states by electrons. Here in the recoil region the peak values of present and first Born and 3P-state results [29] are about double results of the other compared results. The binary peak values of the present results slightly shifted right from other compared results.

In Figure 1(b), the peak value of present and first Born results are lower than the hydrogenic ground state experimental results [2], hydrogenic metastable 3P-state results [29] and hydrogenic metastable 3S-state results [27]. Also the peak values shifted slightly to the higher ejected angle near about $\theta_{1}=72^{\circ}$. The peak pattern of the present result shows exactly similar conduct with the hydrogenic ground state BBK results [11] with slight shift. 


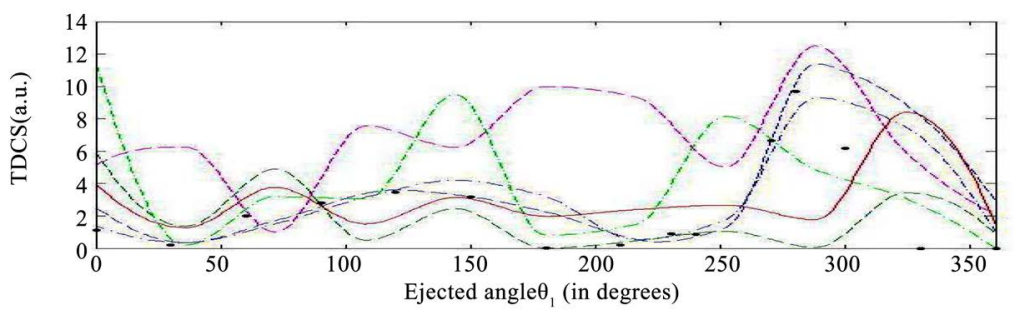

(a)

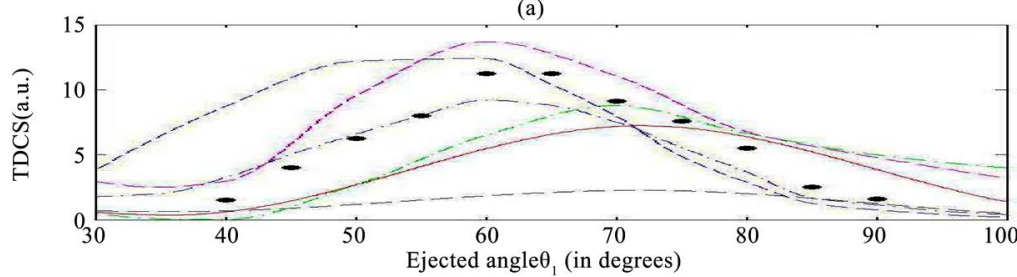

(b)

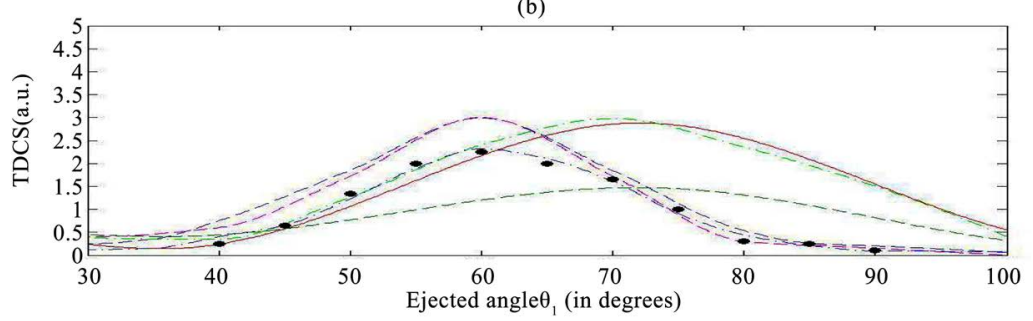

(c)

Figure 1. Triple-differential cross sections (TDCS) with versus ejected electron angle $\theta_{1}$ for atomic hydrogen by electron energy $250 \mathrm{eV}$ with (a) $E_{1}=5 \mathrm{eV}$ and $\theta_{2}=3^{\circ}$, (b) $E_{1}=50 \mathrm{eV}$ and $\theta_{2}=15^{\circ}$, (c) $E_{1}=50 \mathrm{eV}$ and $\theta_{2}=25^{\circ}$. Theory: Continuous curve (Red) illustrate Present result, Dash curve (Black) exhibit Present First Born results, Dash dotted curve (Green) display 3P-state result [29], Dash curve (Magenta) expose 3S-state result [27] and Dash curve (Blue) demonstrate Hydrogenic ground state Second Born results [16], Dash dotted curve (Blue) reveal Hydrogenic ground state BBK model [11] and Round indicated Hydrogenic ground state experiment [2] (multiplied by 0.00224).

In Figure 1(c), our present result and hydrogenic 3P-state result exits in almost same position with similar peak height. The peak magnitude is the highest among other compared results [2] [11].

In Figure 2(a), our present results show a good agreement with 2S-state [18] and $3 S$-state [27] metastable results The present TDCS curve display two fall and two peak in recoil region and one fall two peak in binary region while metastable 3S-state result exhibit one fall and one peak in recoil region and one peak in binary region. The present results and $2 \mathrm{~S}$-state results show one prominent peak in recoil region.

In Figure 2(b), our present study results exhibit same peak pattern with metastable $3 S$-state [27] and 2S-state [18] results whereas the present results and metastable 3P -state results [29] show opposite peak pattern at higher ejected angle about $\theta_{1}=288^{\circ}$.

In Figure 2(c), our present TDCS curve depict a very interesting results. It expressed two falls in recoil region and two peak in binary region. The present result is closer to the 3S-state result [27]. The present result shows a bit different from 3P state results [29]. 


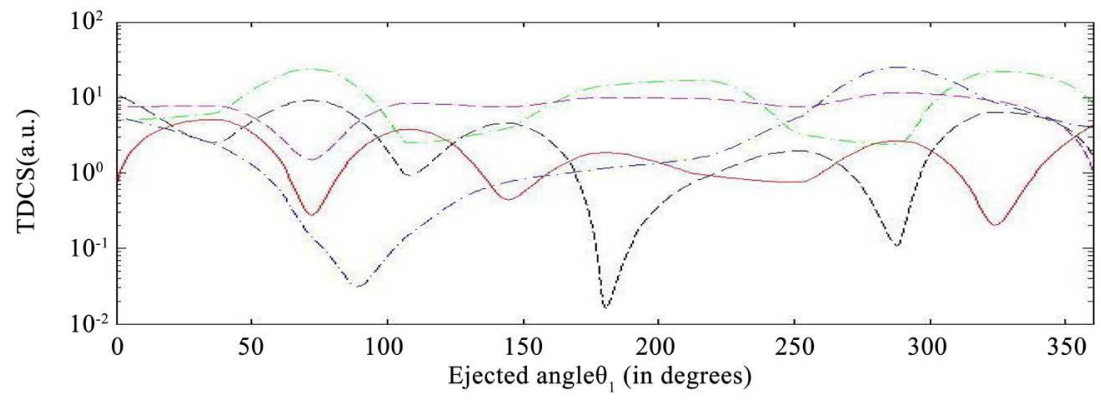

(a)

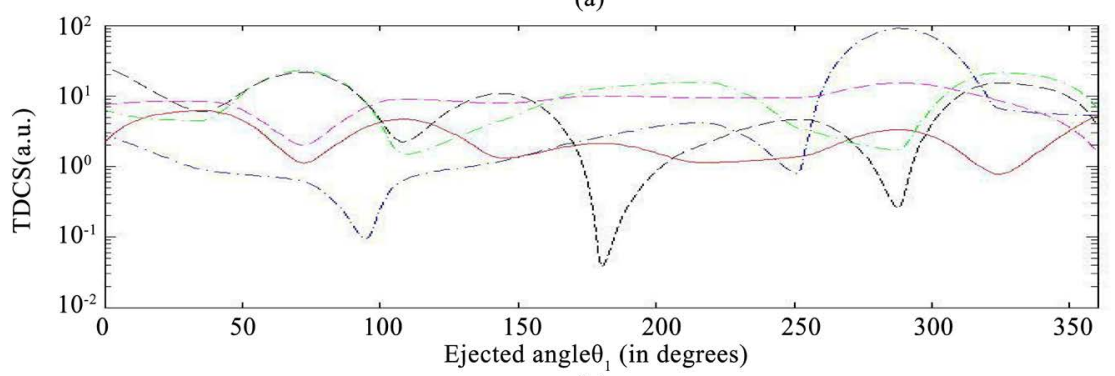

(b)

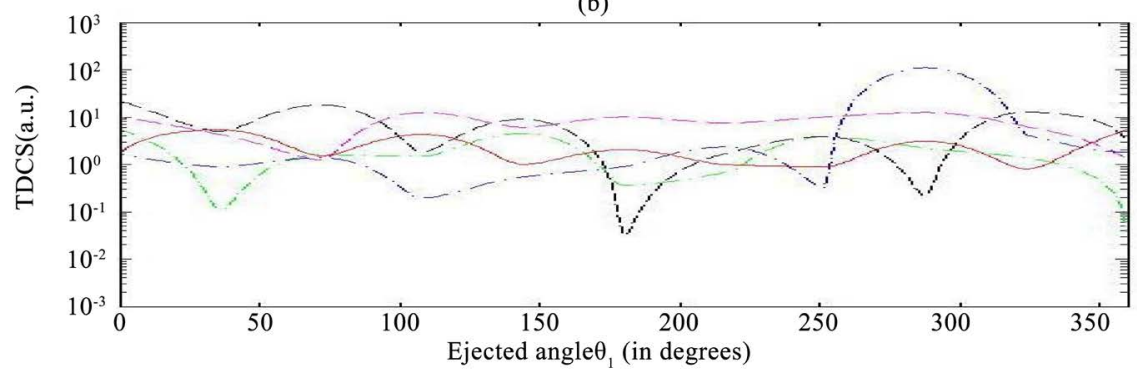

(c)

Figure 2. Triple-differential cross sections (TDCS) versus ejected electron angle $\theta_{1}$ for atomic hydrogen by electron energy $E_{i}=250 \mathrm{eV}$ with ejected electron energy with $E_{1}=5 \mathrm{eV}$ and (a) $\theta_{2}=5^{\circ}$, (b) $\theta_{2}=7^{\circ}$ (c) $\theta_{2}=9^{\circ}$. Theory: Continuous curve (Red) illustrate Present result, Dash curve (Black) exhibit Present First Born results, Dash dotted curve (Green) display hydrogenic 3P-state result [29], Dash curve (Magenta) expose hydrogenic3S-state result [27] and Dash dotted curve(Blue) demonstrate hydrogenic $2 S$-state result [18].

In Figure 3(a), we note that the magnitude of the present and first Born results (black) are lesser than 3S-state [27] and 3P-state [29] results. In this figure, the present result gives a short lobe where $2 \mathrm{~S}$-state [18] result show a very sharp peak at ejected angle about $252^{\circ}$.

In Figure 3(b), the present and first Born results (black) represent similar peak pattern comparing with the 3S-state [27] hydrogenic results whereas the present TDCS curve and first Born curve provide dissimilar peak in the binary region.

In Figure 3(c), the present results and first Born results (black) provide exactly similar behavior as the $3 \mathrm{~S}$ state [27] results but show a gross difference with the results of 2S-state [18] both in recoil and binary region. The present TDCS curve displays a sharp lobe whereas $2 \mathrm{~S}$-state hydrogenic results show a opposite peak at ejected angle near about $\theta_{1}=72^{\circ}$. 


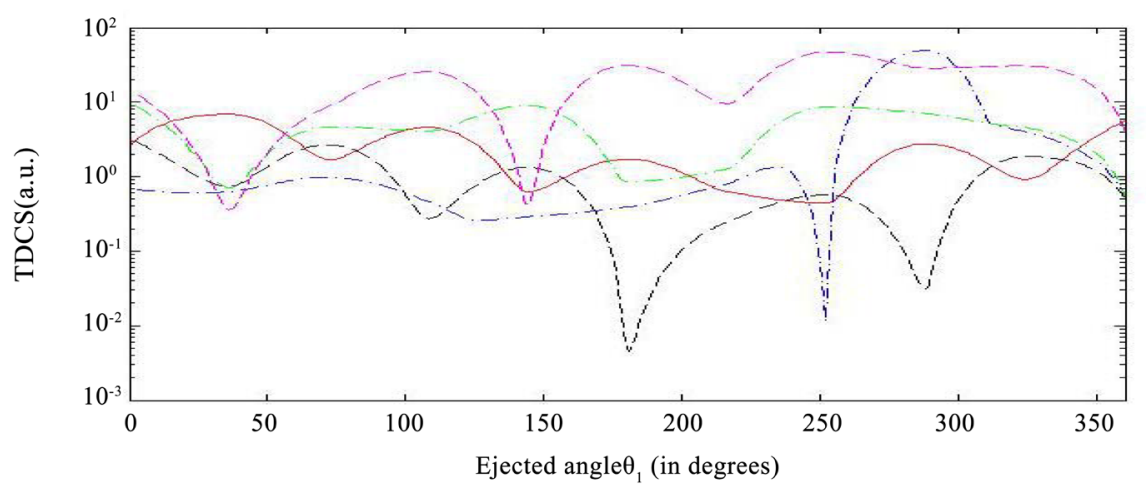

(a)

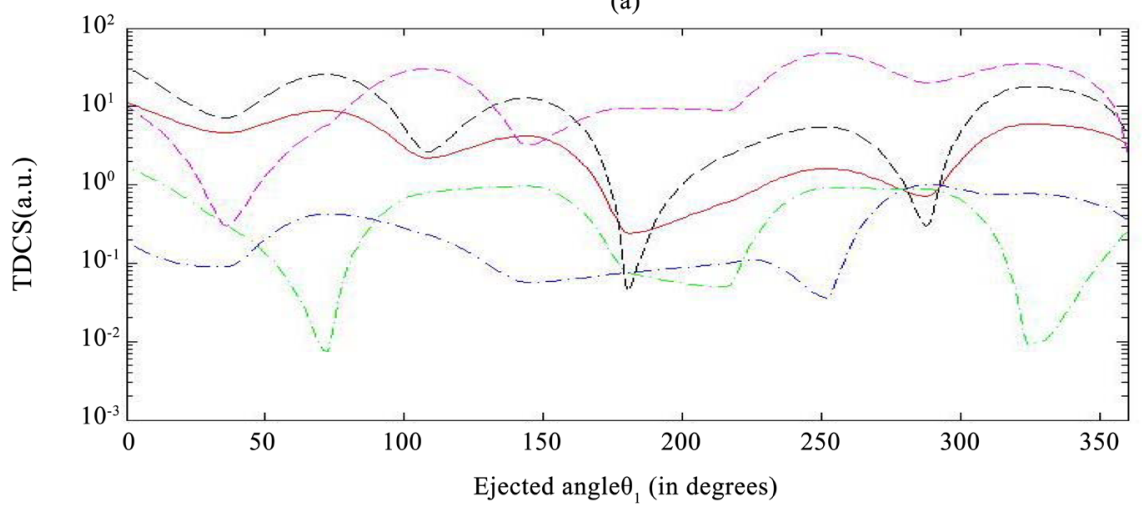

(b)

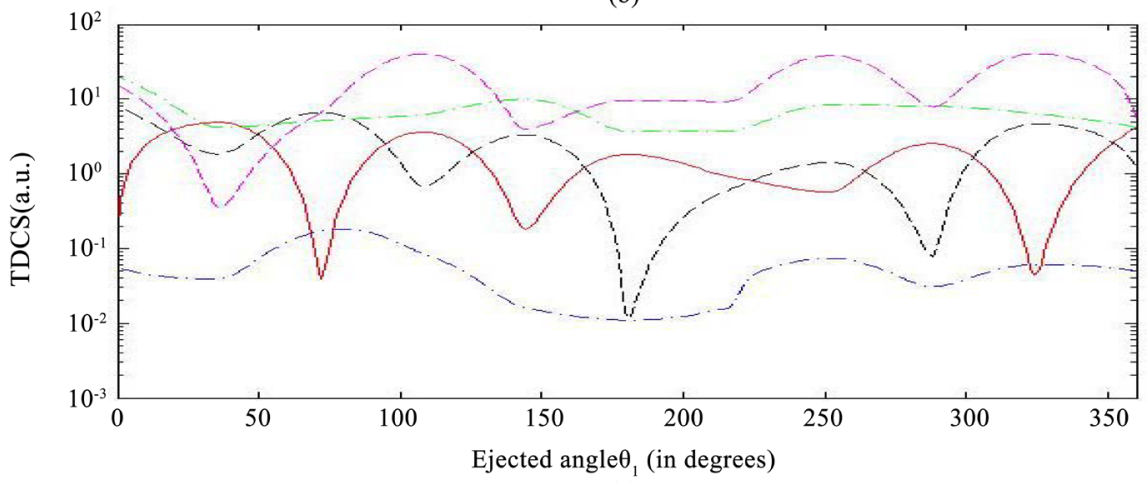

(c)

Figure 3. Triple-differential cross sections (TDCS) versus ejected electron angle $\theta_{1}$ for atomic hydrogen by electron energy $E_{i}=250 \mathrm{eV}$ with ejected electron energy with $E_{1}=5 \mathrm{eV}$ and (a) $\theta_{2}=11^{\circ}$, (b) $\theta_{2}=15^{\circ}$, (c) $\theta_{2}=20^{\circ}$ Theory: Continuous curve (Red) illustrate Present result, Dash curve (Black) exhibit Present First Born results, Dash dotted curve (Green) display hydrogenic 3P-state result [29], Dash curve (Magenta) expose hydrogenic3S-state result [27] and Dash dotted curve(Blue) demonstrate hydrogenic 2S-state result [18].

Finally, Metastable 3d-state is an excited state of an atom or other system with a longer lifetime than the other excited states. The lifetime of $3 \mathrm{~d}$ state of hydrogen atom is $2.3 \times 10^{-7}$. The lifetime of excited state is given by [35]. However, it has a shorter lifetime than the stable ground state. The peak structure of the present results show good qualitative agreement with compared result in the recoil region but show somewhat disagreement in the binary region. This may be 
Table 1. Triple differential cross section (TDCS) for electron impact Ionization of $\mathrm{H}$ (3d) by incident electron are distinguished with $3 \mathrm{P}$-state, $3 \mathrm{~S}$-state \& $2 \mathrm{~S}$-state results where the incident energy is $250 \mathrm{eV}$, the scattering angle is $\theta_{2}=9^{\circ}$ and the ejected electron energy is $E_{1}=5 \mathrm{eV}$.

\begin{tabular}{ccccc}
\hline Ejected angle $\left(\theta_{1}\right)$ & $2 \mathrm{~S}$ & $3 \mathrm{~S}$ & $3 \mathrm{P}$ & $3 \mathrm{~d}$ \\
\hline 0 & 1.6501 & 5.0059 & 11.8679 & 1.6989 \\
36 & 0.9001 & 6.2634 & 0.4252 & 5.3959 \\
72 & 1.3875 & 1.2531 & 2.1485 & 1.5032 \\
108 & 0.1952 & 7.5195 & 3.5834 & 4.3023 \\
144 & 0.5401 & 6.2167 & 9.7624 & 1.0020 \\
180 & 0.8989 & 10.0276 & 0.3155 & 2.0506 \\
216 & 2.3450 & 9.5268 & 0.7248 & 0.9956 \\
252 & 0.3201 & 10.0284 & 8.4297 & 0.8921 \\
288 & 110.00 & 12.5360 & 5.0455 & 3.1015 \\
324 & 4.1569 & 6.2681 & 1.7845 & 0.8012 \\
360 & 1.7890 & 1.2506 & 0.0850 & 5.4074 \\
\hline
\end{tabular}

happened due to the change of the hydrogenic metastable states ionization by electrons. It is remarked that, the peak pattern of the energy spectrum as obtained from our present study is closer to the compared results [27] [29] in some cases and again sometimes different. It may be happened due to the change of atomic state.

Here a table (please see Table 1 ) of comparison results for ionization of hydrogenic $2 \mathrm{~S}$-state, $3 \mathrm{~S}$-state and $3 \mathrm{P}$-state atoms by electron is presented.

\section{Conclusion}

The present estimation reveals imaginable additional formation of the cross-section curves for intermediate momentum transfer in the ionization of the hydrogen atoms in the hydrogenic metastable $3 \mathrm{~d}$-state at $250 \mathrm{eV}$ electron impact energy. It is remarked that, the implementation of the final state wave function of Das and Seal [15] yields good qualitative agreement with hydrogenic ground state as well as metastable $2 \mathrm{~S}$-state, $3 \mathrm{~S}$-state and $3 \mathrm{P}$-state results for qualitative enhancement. It is a new work. New empirical outcomes for ionization of metastable 3d-state hydrogen atoms by electrons will be valuable and originate a novel dimension in order to perceive the study of ionization problems.

\section{Acknowledgements}

The computational works are executed in the Simulation Lab of Department of Mathematics, Chittagong University of Engineering and Technology, Chittagong-4349, Bangladesh. 


\section{Conflicts of Interest}

The authors declare no conflicts of interest regarding the publication of this paper.

\section{References}

[1] Bethe, H. (1930) Zur Theorie des Durchgangs schneller Korpuskularstrahlen durch Materie. Annalen der Physik, 397, 325-400. https://doi.org/10.1002/andp.19303970303

[2] Ehrhardt, H., Knoth, G., Schlemmer, P. and Jung, K. (1986) Differential Cross Sections of Direct Single Electron Impact Ionizatio. Zeitschrift für Physik D Atoms, Molecules and Clusters, 1, 3-32. https://doi.org/10.1007/BF01384654

[3] Ehrhardt, H., Knoth, G., Schlemmer, P. and Jung, K. (1985) Absolute H(e, 2e)p Cross Section Measurements: Comparison with First and Second Order. Physics Letters A, 110, 92-94. https://doi.org/10.1016/0375-9601(85)90326-3

[4] Ehrhardt, H. and Roder, J. (1997) In: Whelan, C.T. and Walters, H.R.J., Eds., Coincidence Studies of Electron and Photon Impact Ionization, Plenum, New York, $1-10$.

[5] Das, J.N. (1990) Momentum-Space Analysis of Scattering States with Possible Application to Atomic Ionization. Physical Review A, 42, 1376. https://doi.org/10.1103/PhysRevA.42.1376

[6] Das, J.N. and Seal, S. (1993) Symmetric Scattering Ine $\pm-H$ Ionization Collisions. Pramana, 40, 253-258. https://doi.org/10.1007/BF02900191

[7] Das, J.N. and Dhar, S. (1999) Energy Spectrum of Ejected Electrons in Ionization of Hydrogen Atoms by Electrons. Pramana, 53, 869-875.

https://doi.org/10.1007/s12043-999-0121-9

[8] Joachain, J.C. and Piraux, B. (1986) Theory of Coplanar Asymmetric(e,2e) Reactions in Helium. Molecular Physics, 17, 261.

[9] Byron, W.F., Joachen, J.C. and Piraux, B. (1980) Triple Differential Cross Sections for the Ionization of Atomic Hydrogen by Fast Electrons: A Second Born Treatment. Journal of Physics B: Atomic, Molecule and Optical Physic, 13, L673.

[10] Byron, W.F., Joachain, J.C. and Piraux, B. (1986) Theory of Coplanar Asymmetric (e, 2e) Reactions in Helium. Journal of Physics B: Atomic, Molecule and Optical Physic, 19, 120.

[11] Brauner, M., Briggs, J.C. and Klar, H. (1989) Triply-Differential Cross Sections for Ionisation of Hydrogen Atoms by Electrons and Positrons. Journal of Physics B: Atomic, Molecule and Optical Physic, 22, 2265. https://doi.org/10.1088/0953-4075/22/14/010

[12] Brauner, M. and Briggs, J.C. (1986) Ionisation to the Projectile Continuum by Positron and Electron Collisions with Neutral Atoms. Journal of Physics B: Atomic, Molecule and Optical Physic, 19, L325. https://doi.org/10.1088/0022-3700/19/9/006

[13] Brauner, M., Briggs, J.C. and Klar, H. (1991) Structures in Differential Cross Sections for Positron Impact Ionization of Hydrogen. Journal of Physics B: Atomic, Molecule and Optical Physic, 24, 2227. https://doi.org/10.1088/0953-4075/24/8/030

[14] Berakder, J. and Klar, H. (1993) Structures in Triply and Doubly Differential Ionization Cross Sections of Atomic Hydrogen. Journal of Physics B: Atomic, Molecule and Optical Physic, 26, 3891. https://doi.org/10.1088/0953-4075/26/21/023

[15] Das, J.N. and Seal, S. (1993) Electron-Hydrogen Atom Ionization Collisions at In- 
termediate (510-2010) and High( $\approx 2010)$ Energies. Physical Review A, 47, 2978.

[16] Dal Capppello, C., Haddadou, A., Menas, F. and Roy, A.C. (2011) The Second Born Approximation for the Single and Double Ionization of Atoms by Electrons and Positrons. Journal of Physics B: Atomic, Molecular and Optical Physics, 44, Article ID: 015204. https://doi.org/10.1088/0953-4075/44/1/015204

[17] Berakdar, J., Engelns, A. and Klar, H. (1996) Oriented and Aligned Two Electron Continue. Journal of Physics B: Atomic, Molecular and Optical Physics, 29, 1109. https://doi.org/10.1088/0953-4075/29/5/019

[18] Dhar, S. (1996) Electron Impact Ionisation of Metastable 2S-State Hydrogen Atoms. Australian Journal of Physics, 49, 937-944. https://doi.org/10.1071/PH960937

[19] Das, J.N. and Dhar, S. (1996) Symmetric Scattering in Electron and Positron Impact Ionization of Metastable 2S-State Hydrogen Atom. Pramana-Journal of Physics, 47, 263-269.

[20] Das, J.N. and Dhar, S. (1998) Calculation of Triple Differential Cross-Sections of K-Shell Ionization of Medium-Heavy Atoms by Electrons for Symmetric Geometry. Pramana, 51, 751-756. https://doi.org/10.1007/BF02832607

[21] Vučič, S., Potvliege, R.M. and Joachain, C.J. (1987) Second Born Triple-Differential Cross Sections for the Coplanar Asymmetric Ionization of $\mathrm{H}(2 \mathrm{~S})$ by Fast Electrons. Physical Review A, 35, 1446-1449.

[22] Qi, Y.Y., Ning, L.N., Wang, J.G. and Qu, Y.Z. (2013) Plasma Effect on Fast-Electron-Impact Ionization from 2p State of Hydrogen-Like Ions. Physics of Plasmas, 20, Article ID: 123301. https://doi.org/10.1063/1.4833616

[23] Dhar, S. and Nahar, N. (2015) Energy Spectrum of Ejected Electrons of H(2P) Ionization by Electrons in Coplanar Asymmetric Geometry. American Journal of Modern Physics, 4, 132.

[24] Dhar, S. and Nahar, N. (2014) Ionization of Metastable 2P-State Hydrogen Atoms by Electron Impact for Coplanar Asymmetric Geometry. Open Journal of Microphysics, 4, 46-53. https://doi.org/10.4236/ojm.2014.44007

[25] Dhar, S. and Nahar, N. (2015) Electron Impact Ionization of Metastable 2P-State Hydrogen Atoms in the Coplanar Geometry. Results in Physics, 5, 3-8.

https://doi.org/10.1016/j.rinp.2014.11.001

[26] Dhar, S., Noor, T. and Chowdhury, F.S. (2015) Electron Impact Ionization of Metastable 3S-State Hydrogen Atoms by Electrons in Coplanar Geometry. American Journal of Modern Physics, 4, 261-266.

[27] Noor, T. and Chowdhury, F.S. (2016) Calculation of Triple Differential Cross Sections for the Ionization of $\mathrm{H}(3 \mathrm{~S})$ by Electron Impact. American Journal of Modern Physics, 5, 162-171.

[28] Dhar, S., Akter, S. and Nahar, N. (2016) First Born Triple Differential Cross-Section for Ionization of $\mathrm{H}(3 \mathrm{P})$ by Electron Impact in the Asymmetric Coplanar Geometry. Open Journal of Microphysics, 6, 15-23.

[29] Dhar, S., Akter, S., Saha, A. and Nahar, N. (2017) Triple Differential Cross Sections for Ionization of Metastable 3P-State Hydrogen Atoms by Electrons. IOSR Journal of Applied Physics, 9, 21-29.

[30] Das, J.N. and Chakraborty, S. (1985) Atomic Inner-Shell Ionization. Physical Review $A$, 32, 176-180. https://doi.org/10.1103/PhysRevA.32.176

[31] Das, J.N. and Dhar, S. (1998) Energy Spectrum of Scattered Electrons in K-Shell Ionization of Medium to Heavy Atoms by Relativistic Electrons. Journal of Physics B: Atomic, Molecular and Optical Physics, 31, 2355. 
https://doi.org/10.1088/0953-4075/31/10/021

[32] Dhar, S. (2008) The Energy Spectrum of Scattered Particles in the K-Shell Ionization of Medium Heavy Atoms by Relativistic Electrons and Positrons with Exchange Effects. Journal of Physics B: Atomic, Molecular and Optical Physics, 41, Article ID: 155204. https://doi.org/10.1088/0953-4075/41/15/155204

[33] Jones, S. and Madison, D.H. (2000) Ionization of Hydrogen Atoms by Fast Electrons. Physical Review A, 62, Article ID: 042701. https://doi.org/10.1103/PhysRevA.62.042701

[34] Jones, S. and Madison, D.H. (2002) Scaling Behavior of the Fully Differential Cross Section for Ionization of Hydrogen Atoms by the Impact of Fast Elementary Charged Particles. Physical Review A, 65, Article ID: 052727.

https://doi.org/10.1103/PhysRevA.65.052727

[35] Tenneyson, J. (2011) Astronomical Spectroscopy: An Introduction to the Atomic and Molecular Physics of Astronomical Spectra. 2nd Edition, World Scientific Publishing, Singapore. https://doi.org/10.1142/7574

[36] Lewis, R.R. (1956) Potential Scattering of High-Energy Electrons in Second Born Approximation. Physical Review, 102, 537. 